\title{
Forschung ist Zukunft!
}

Den Ausgangspunkt der modernen Stoffwechselforschung bildet insbesondere die Arbeit von Antoine Laurent de Lavoisier (1743 - 1794), in der erkannt wurde, dass der mit der Atmung zugeführte Sauerstoff im Körper zur Verbrennung kohlenstoff- und wasserstoffreicher Verbindungen dient und dass der Sauerstoffverbrauch durch die körperliche Aktivität eine Steigerung erfährt. Seit dieser Zeit gab und gibt es eine Vielzahl von Studien, die sich mit dem Stoffwechsel und seinen anorganischen und organischen Substraten beschäftigt.

So ist diese Thematik auch in der Sport- und Präventivmedizin von brandaktueller Bedeutung, weil auch die Wirtschaft zunehmend erkannt hat, dass stoffwechsel-modifizierende Faktoren in Form von Nahrungsergänzungsmitteln einen äußerst lukrativen Bereich darstellen. In der aktuellen Ausgabe der Zeitschrift sport- und präventivmedizin greifen vier Artikel diese Thematik auf, wobei auch im ersten Anblick ungewöhnliche Ansätze präsentiert werden, dass zum Beispiel die Konsumation von Genussmitteln - im konkreten Fall ist es Bier - unter stoffwechselspezifischen Aspekten betrachtet wird.

Diese aktuelle Ausgabe ist aber auch dem Begründer der Zeitschrift gewidmet - DDDr. Ludwig Prokop - der vor 40 Jahren das Österreichische Journal für Sportmedizin gegründet hat, welches eine „Runderneuerung“ und einen Relaunch erfahren hat und seit Jänner 2009 mit einem erweiterten Redaktionsteam, einer neuen Chefredaktion und in Kooperation mit dem Springer-Verlag als sportund präventivmedizin herausgegeben wird.

DDDr. Ludwig Prokop hat sich mittlerweile aus dem öffentlichen Leben zurückgezogen; seine Lebenseinstelllung in Bezug auf Arbeitsstress und Entspannung oder Sport und Regeneration möge für unseren oftmals hektischen Alltag Vorbildwirkung haben.

Wir gratulieren Herrn DDDr. Ludwig Prokop ganz herzlich zum 90. Geburtstag und wünschen ihm weiterhin eine schöne Zeit im Kreise seiner Familie und Freunde.

Wir wünschen Ihnen, sehr geehrte Leser und Leserinnen, wieder viel Spaß und Erkenntnisgewinn beim Durchlesen der aktuellen Ausgabe!

\section{Univ.-Prof. Dr. Norbert Bach}

Herausgeber

\section{Dr. Piero Lercher}

Editor in Chief

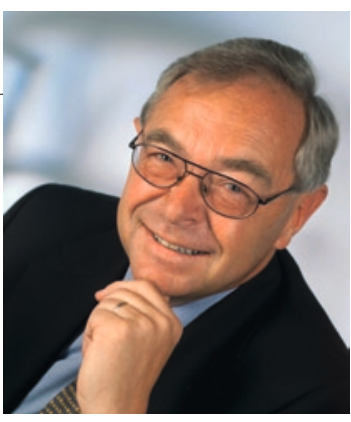

HERAUSGEBER

Univ.-Prof.

Dr. Norbert Bachl

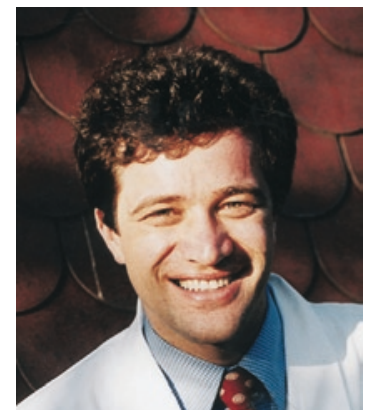

EDITOR IN CHIEF

Dr. Piero Lercher 